\title{
Correction to: Quantification of Inkjet-Printed Pharmaceuticals on Porous Substrates Using Raman Spectroscopy and Near-Infrared Spectroscopy
}

\author{
Magnus Edinger, ${ }^{1}$ Laura-Diana Iftimi, ${ }^{1}$ Daniel Markl, ${ }^{2,3}$ Mohammed Al-Sharabi, ${ }^{4}$ Daniel Bar-Shalom, ${ }^{1}$ \\ Jukka Rantanen, ${ }^{1}$ and Natalja Genina ${ }^{1,5}$
}

Published online 26 June 2019

\section{Correction to: AAPS PharmSciTech (2019) 20:207 \\ https://doi.org/10.1208/s12249-019-1423-y}

Mohammed Al-Sharabi's affiliation was incorrect at the time of publishing. The updated affiliation appears below.

${ }^{1}$ Department of Pharmacy, Faculty of Health and Medical Sciences, University of Copenhagen, Copenhagen, Denmark.

2 Strathclyde Institute of Pharmacy and Biomedical Sciences, University of Strathclyde, Glasgow, UK.

3 EPSRC Centre for Innovative Manufacturing in Continuous Manufacturing and Crystallisation, University of Strathclyde, Glasgow, UK.

4 Department of Chemical Engineering and Biotechnology, University of Cambridge, Philippa Fawcett Drive, CB3 0AS Cambridge, UK.

Publisher's Note Springer Nature remains neutral with regard to jurisdictional claims in published maps and institutional affiliations.

The online version of the original article can be found at https:// doi.org/10.1208/s12249-019-1423-y

\footnotetext{
${ }^{1}$ Department of Pharmacy, Faculty of Health and Medical Sciences, University of Copenhagen, Universitetsparken 2, 2100, Copenhagen, Denmark.

${ }^{2}$ Strathclyde Institute of Pharmacy and Biomedical Sciences, University of Strathclyde, Glasgow, UK.

${ }^{3}$ EPSRC Centre for Innovative Manufacturing in Continuous Manufacturing and Crystallisation, University of Strathclyde, Glasgow, UK.

${ }^{4}$ Department of Chemical Engineering and Biotechnology, University of Cambridge, Philippa Fawcett Drive, CB3 0AS, Cambridge, UK.

${ }^{5}$ To whom correspondence should be addressed. (e-mail: natalja.genina@sund.ku.dk)
} 